\title{
The association between the adaptive/maladaptive personality dimensions and emotional regulation
}

\author{
Sohrab Amiri', Amir Ghasemi Navab² \\ ${ }^{1}$ Urmia University, Faculty of Literature and Humanities, Department of Psychology, Urmia, Iran \\ ${ }^{2}$ Allameh Tabatabaei University, Tehran, Iran \\ Neuropsychiatria i Neuropsychologia 2018; 13, 1: 1-8
}

Address for correspondence:

Ph.D. Sohrab Amiri

Urmia University

Urmia, Iran

e-mail: amirysohrab@yahoo.com

\begin{abstract}
This study investigates the associations between the big five personality dimensions, dark personality traits, and the brief form of the Personality Inventory for DSM-5 with Emotion regulation difficulty dimensions. Data was obtained in a sample of normal population $(N=461)$. Results showed the associations between neuroticism and openness to experience from the big five personality dimensions and emotion regulation difficulty $(p<0.01)$. Machiavellianism and psychopathy from dark traits was associated with emotion regulation difficulty $(p<0.01)$. Negative affectivity, detachment, antagonism, disinhibition, and psychoticism showed positive associations with emotion regulation difficulty $(p<0.01)$. The results of this study expand the understanding of adaptive/maladaptive personality dimensions and indicate how adaptive and maladaptive personality dimensions could explain emotion regulation difficulty.
\end{abstract}

Key words: emotion regulation difficulty, dark trait, big five, maladaptive personality.

\section{Introduction}

Emotions are psycho-physiological phenomena that affect our thoughts and experiences about the world (Bradley and Lang 2000). It is believed that emotional systems are shaped by evolution (Panksepp 2012). The emotions include the range of responses from severe to mild, and from positive to negative (Kring and Sloan 2010). In general, contemporary theories of emotion emphasise the role and importance of emotions in providing physiological, behavioural, and motor responses, facilitating decision making, and promoting memory for important incidents and interpersonal interactions (Gross and Thompson 2007). Given what has been said, emotions play an important role in different aspects of life, such as adaptation to life changes and stressful events. As was proven, emotion regulation difficulties can lead to various psychological disorders (Surawy, Hackmann, Hawton and Sharpe 1995; Gross and Levenson 1997; Ehlers and Clark 2000; Ali et al. 2000; Cramer, Gallant and Langlois 2005; Corstorphine 2006; Woolfolk and Allen 2007; d'Acremont and Van der Linden 2007; Betts, Gullone and
Allen 2009; Azad-Marzabadi and Amiri 2017). Also, the results of the studies show the role of emotion in the range of psychological activities, including attention, memory, and physiological dimensions (Vuilleumier 2005).

It seems that emotion regulation difficulties are related to different personality dimensions. One of the personality models that has attracted so much attention in recent years is the theory of dark traits. In order to achieve long-term goals and adapt to social norms, people must learn how to regulate their emotions (Dahl 2004). Emotion regulation refers to the processes through which individuals influence the way of expressing and experiencing emotions (Gross 1998). Therefore, emotion regulation is an essential component for the achievement of optimal social performance (Macklem 2008).

The dark triad traits theory contains three personality bridges: narcissism, Machiavellianism, and psychopathy; although these personality traits are related to each other, they are at the same time independent of each other (Paulhus and Williams 2002). Narcissism refers to exaggerated feelings of oneself, and an inflated sense of one's own importance, merit, mastery, 
and superiority (Twenge and Campbell 2003). Psychopathy includes dimensions such as impulsivity, lack of empathy towards others, and lack of anxiety (Paulhus and Williams 2002). Machiavellianism refers to exploiting others in one's interest, lack of intimacy, cruelty, and hegemonic behaviour (Jakobwitz and Egan 2005).

Lack of emotion and emotional experiences is one of the main characteristics in individuals with dark traits (Jonason, Lyons, Bethell and Ross 2012). Research on dark traits has shown that psychopathy is negatively associated with emotional experiences, so individuals with high psychopathy tend to pay less attention to their emotions (Malterer, Glass and Newman 2008). It seems that the psychopathy trait has emotional experience deficiencies, which lead to insensitivity to emotions (Patrick and Lang 1999). There are some limitations in examining the relationships between the dark dimensions of personality and excitement: addressing only one of the dark traits (Brook and Kosson 2013) and that some aspects of emotions, such as empathy, are more closely investigated in relation to dark traits (Shamay-Tsoory, Aharon-Peretz and Perry 2009). What is clear is that the psychopathic trait has a close relationship with the dimensions of the negative emotions (Wai and Tiliopoulos 2012), and emotional defects play a prominent role in dark traits, although various dimensions of emotion and especially emotion dysregulation have not yet been fully studied in relation to these traits (Ali, Amorim and Chamorro-Premuzic 2009).

With regard to various concerns about personality disorders since the third edition of the Diagnostic and Statistical Manual of mental disorders (DSM-III; American Psychiatric Association 1980), such as the lack of experimental cut-off scores, an alternative model of personality disorder (AMPD) in the third section of DSM-5 (American Psychiatric Association 2013) provided, along with classic personality disorder criteria listed in the DSM-5 (which includes the second axis DSM personality disorder criteria IV; American Psychiatric Association 1994). The alternative personality disorder model allows clinical professionals to examine the five main areas of maladaptive personality, including negative affectivity (the frequency and intensity of the experience of the levels of negative emotions), detachment (avoiding social and emotional experiences), antagonism (the behaviours of a person in confronting others), disinhibition (orientation toward immediate satisfaction and impulsive behaviour), and psy- choticism (behaviours and cogitations that are strange and unusual). This model represents a dimensional model of personality pathology, focused on a variety of maladaptive personality traits (Clark 2007; Harkness, McNulty and Ben-Porath 1995; Trull and Durrett 2005; Widiger and Simonsen 2005). But an adaptive personality model that has been widely considered is the big five model of personality.

The Big Five Personality Model is widely used to assess the normal personality dimensions. This personality model includes five factors: conscientiousness, agreeableness, extraversion, intellect/openness, and neuroticism. It has been shown that these personality dimensions have a high stability throughout life (Roberts, Walton and Veichtbauer 2006). Due to the problems of the five-factor model, the Mini International Personality Item Pool (Mini-IPIP) was formed in order to evaluate personality (Donnellan, Oswald, Baird and Lucas 2006). There are many individual differences in the ability to regulate emotions (Cole, Michel and Teti 1994), and different individuals use different emotion regulations strategies that can predispose them to psychological outcomes. These differences have been considered in some personality models (McCrae and Costa 1987). For example, it has been shown that the big five personality dimensions, namely: extraversion and neuroticism, are differently associated with emotion regulation (Dasch, Cohen, Sahl and Gunthert 2008; Mroczek and Almeida 2004; Timmermans, Van Mechelen and Nezlek 2009). Also, the emotion regulation difficulty is associated with some personality disorders mentioned in the Diagnostic and Statistical Manual of Mental Disorders (DSM-5; American Psychiatric Association 2013); for example, associations with borderline personality disorder (Rosenthal et al. 2008; Herr, Rosenthal, Geiger and Erikson 2013) and pathological personality dimensions (Pollock, McCabe, Southard and Zeigler-Hill 2016). Although some studies (Pollock et al. 2016) have studied the emotion regulation difficulty in the pathological aspects of the personality, but these studies have limitations such as merely examining the maladaptive dimensions of personality in relation to the difficulty of emotional regulation, and the adaptive dimensions have not been considered. In general, based on the above, the purpose of the present study was to examine the association between emotion regulation difficulty and the dimensions of adaptive (big five model) and maladaptive (dark traits and Personality Inventory for DSM-5) personality traits. 


\section{Material and methods}

\section{Participants and procedure}

The 461 students were selected from among Bu-Ali university students in Iran. Students were selected from five colleges based on cluster sampling, which included the faculties of Economics, Agriculture, Basic Sciences, Chemistry, and Literature. Research questionnaires were given to the participants to answer. After collecting data, participants who did not have the criteria for entering the study were excluded from the analysis. Inclusion and exclusion criteria were collected through a checklist of self-reporting along with questionnaires. Inclusion criteria were as follows: 1) report of gender; 2) age; 3) non-dependence on any type of substance and smoking; 4) not having cognitive and psychiatric problems; and 5) not having brain damage. Exclusion criteria were: 1) students of other cultures; and 2) participants with overweight and obesity.

\section{Measures}

\section{Emotion regulation difficulty questionnaire}

The questionnaire consists of 36 items and six sub-scales that are scored based on the five-point Likert scale. The subscales include: 1) lack of emotional awareness; 2) lack of emotional clarity; 3) non-acceptance of emotional responses; 4) limited access to emotion regulation strategies; 5) difficulties engaging in goal-directed behaviour; and 6) impulse control difficulties. The emotion regulation difficulty scale has good internal consistency ( $\alpha=0.93$; Gratz and Roemer 2004).

\section{Short Dark Triad (SD3)}

The Short Dark Triad Scale includes 27 items and three subscales. Each of the three subscales in this questionnaire contains nine items, which are used to assess the three personality traits: Machiavellianism, narcissism, and psychopathy. The items in this scale are scored on the basis of participants' agreement or disagreement, considering five-degree Likert scale: from 1 (strongly disagree) to 5 (strongly agree). In Jones and Paulhus's (2014) study the $\alpha$ coefficient range was 0.68 to 0.74 . The researchers performed this scale on different large population samples $(N=768)$. Peer validation was also carried out on 65 university students (Jones and Paulhus 2014). The subscales showed Cronbach's $\alpha$ coefficients 0.70 to 0.80 in cross-validation samples (Jones and Paulhus 2014). The range 0.77 to 0.84 was reported for retest coefficients in a two-week interval (Jones and Paulhus 2011).

\section{Big five personality inventory (short form)}

This questionnaire is designed to measure five main personality dimensions. These personality dimensions include: extraversion, agreeableness, conscientiousness, neuroticism, and openness to experience (Laverdičre, Morin and St-Hilaire 2013). The correlation coefficient of these subscales is reported in the optimal range, but Cronbach's $\alpha$ coefficient has not been reported (Laverdière, Morin and St-Hilaire 2013).

\section{Personality Inventory for DSM-5 (briefform)}

The questionnaire consists of 25 items designed to measure five dimensions of the substitution model of personality disorder, including negative affectivity, detachment, antagonism, disinhibition, and psychoticism in both adults and adolescents. Each subscale contains five items and is scored according to the four-point Likert scale (Krueger, Derringer, Markon, Watson and Skodol 2012). The study of the psychometric properties of this scale obtained the $\alpha$ coefficient range 0.59 to 0.77 (Fossati, Somma, Borroni, Markon and Krueger 2017).

\section{Data analytic strategy}

All data was analysed through SPSS version 22. Bivariate correlations and multiple regressions analysis were performed. In this study, personality variables were considered as independent variables and difficulty of emotional regulation as a dependent variable. Because most of the sub-components of the emotion regulation difficulty were related to different personality dimensions, only one general variable of emotional regulation was introduced into analysis.

\section{Results}

\section{Descriptive statistics}

Of the total participants, 392 were undergraduate students $(84.8 \%)$ and 70 were masters students (15.2\%), 375 participants were female $(81.2 \%)$ and 87 were male $(18.8 \%)$. The students' mean age and standard deviation were 25.05 and 5.88, in males, 21.66 and 4.91 in females, respectively. Bivariate correlations among the study variables are presented in Table 1.

Skew and kurtosis are in conventional range (-1 to 1; George and Mallery 2003) for all of 


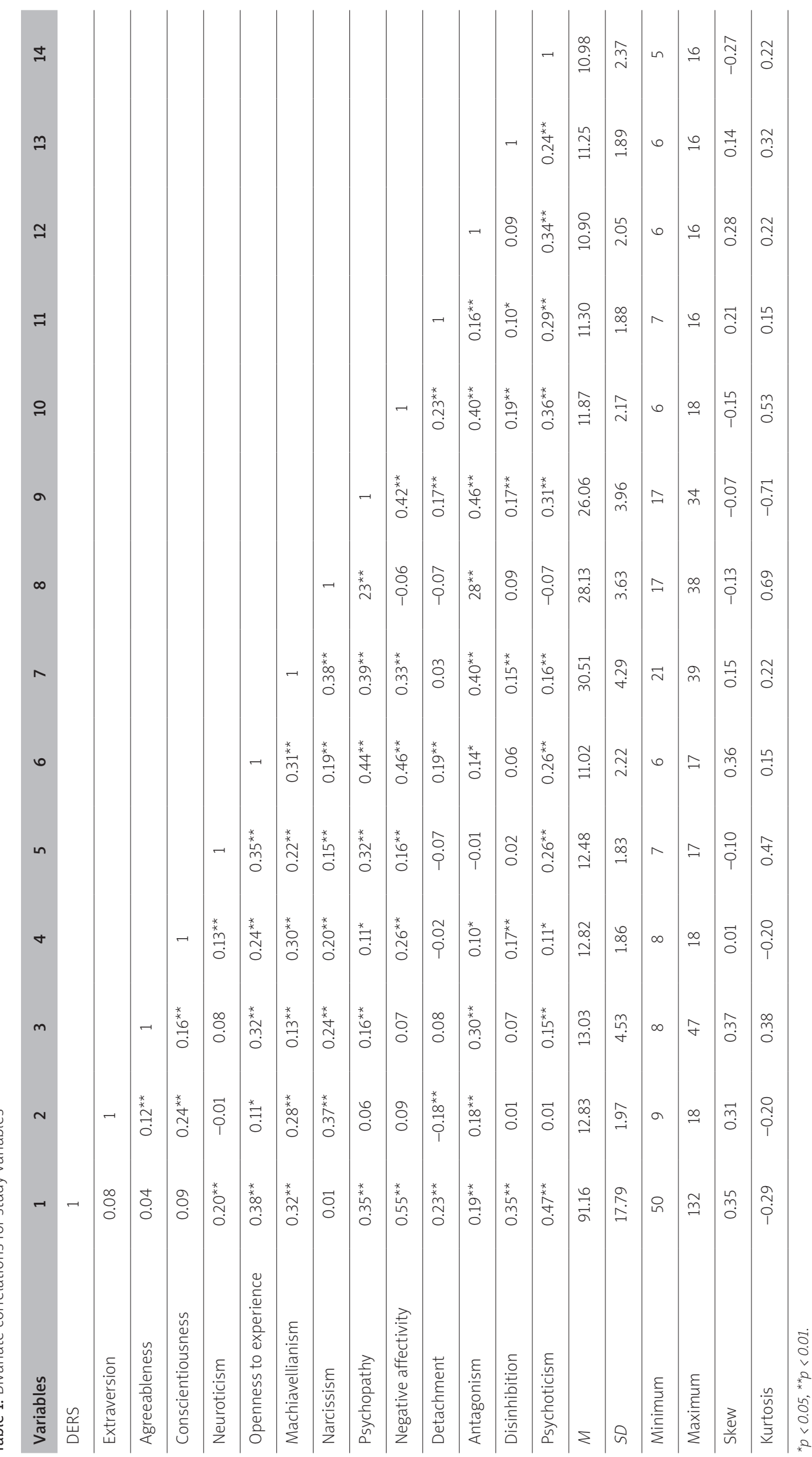




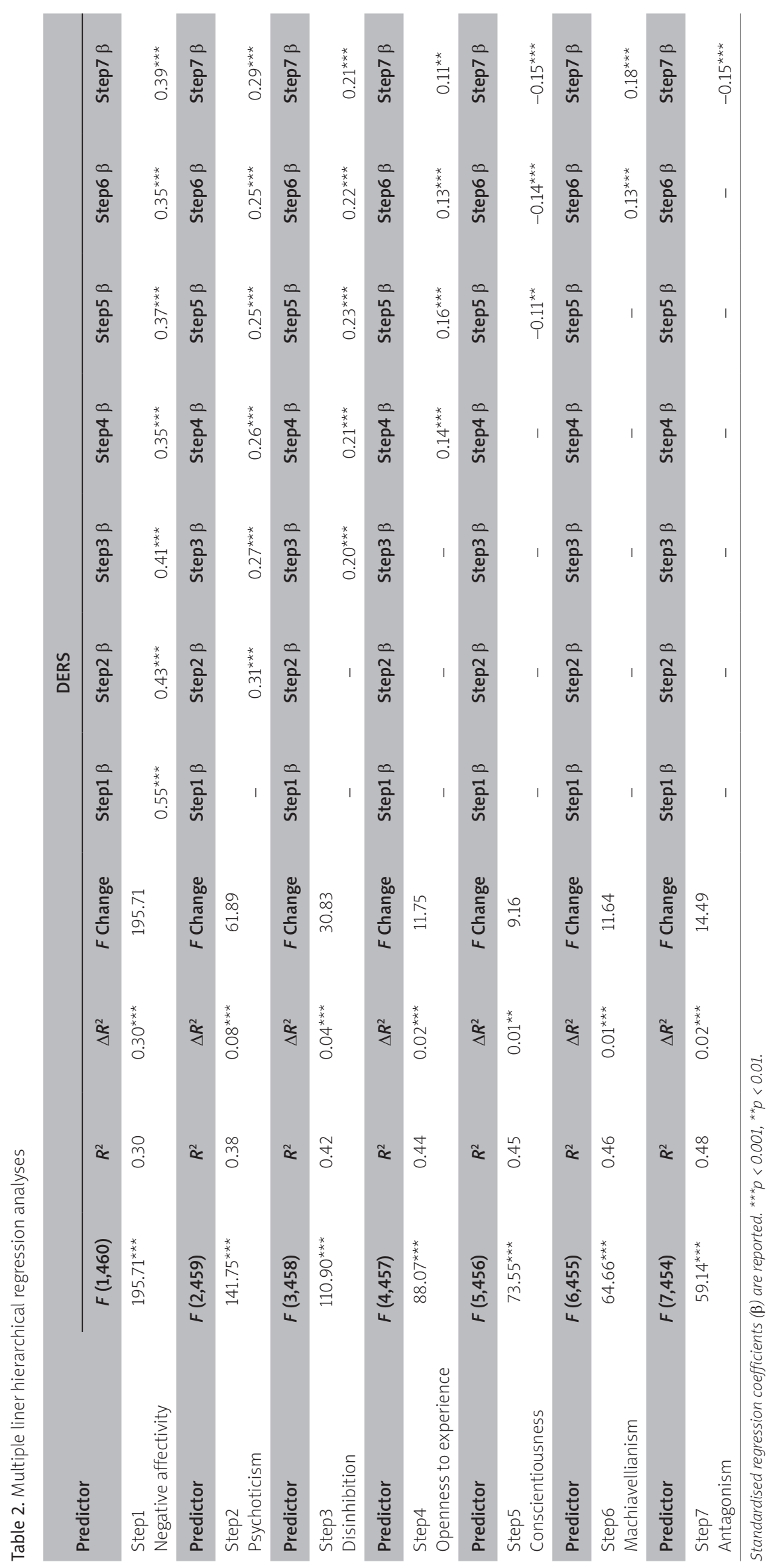


the variables. Bivariate correlations showed that the big five personality dimensions including neuroticism and openness to experience were significantly associated with emotion regulation difficulty ( $p<0.01$; see Table 1$)$. Machiavellianism and psychopathy from the dark traits have positive correlations with emotion regulation difficulty $(p<0.01)$. All dimensions of maladaptive personality include negative affectivity, detachment, antagonism, disinhibition, and psychoticism were positively associated with emotion dysregulation $(p<0.01)$.

Multiple hierarchical regression analysis was conducted to investigate the relationships between adaptive and maladaptive personality dimensions with emotion regulation difficulty (see Table 2). Among the maladaptive dimensions of personality, only negative affectivity, psychoticism, disinhibition, and antagonism were predictive of emotion regulation difficulty. Among the big five personality dimensions, openness to experience and conscientiousness were predictive of emotion regulation difficulty. Among the dark personality traits, only Machiavellianism was a predictor of emotion regulation difficulty. Overall, adaptive and maladaptive personality dimensions explained $48 \%$ of the variance in emotion regulation difficulty. Because of the use of hierarchical regression in this regression, various variables are entered into the model, and therefore variables that previously had a meaningful regression coefficient may lose their meaningful value after the arrival of the new variable entered into the analysis.

\section{Discussion}

The purpose of this study was to investigate the relationship between adaptive and maladaptive personality dimensions with emotion regulation difficulty. Based on this, the findings of this study showed that there were positive associations between emotion regulation difficulty with both adaptive and maladaptive personality dimensions. As expected, the dimensions of the dark traits of personality including Machiavellianism and psychopathy have a positive association with emotion regulation difficulty, and it was a predictor of emotional problems. This finding is consistent with studies that show an emotional defect in individuals with dark traits (Jonason, Lyons, Bethell and Ross 2013; Malterer, Glass and Newman 2008). It can be argued that the emotional deficits that exist in individuals with dark traits express the fundamental component of the emotion regulation difficulty in these individuals. On the other hand, current findings are closely related to a study that showed that Machiavellianism and psychopathy are closely related to the deficiency in empathy and emotional expression (Ali, Amorim and Chamorro-Premuzic 2009). Behavioural genetics research on these relationships show that almost all the significant correlations in these cases are ascribable to common genetic factors (Jonason, Webster, Schmitt, Li and Crysel 2012).

Another goal of this research was to evaluate the associations between emotion regulation difficulty and maladaptive personality traits, including negative affectivity, detachment, antagonism, disinhibition, and psychoticism. As the results showed, there was a high correlation between all aspects of maladaptive personality with the emotion regulation difficulty. Accordingly, the negative affectivity component had the highest power of prediction of emotion regulation difficulty. This finding is in line with a study that showed the association between emotional regulation difficulty and maladaptive personality (Pollock et al. 2016). Also, in the previous study (Pollock et al. 2016), the negative affectivity component had the highest correlation with excitement emotion regulation difficulty, accordingly, and the findings of this study can include valuable insights on this association.

Another finding of the present study, which should be noted, is that the big five factor model of personality, known as adaptive personality theory, has no association with emotion regulation difficulty, except in the two components of neuroticism and openness to experience. Therefore, the result of this study about the associations of the big five personality model with emotion regulation difficulty is in line with previous studies (Bolger and Schilling 1991; Dasch et al. 2008; Mroczek and Almeida 2004; Timmermans, Van Mechelen, and Nezlek 2009). But it should be noted that unlike previous studies, the present study uses a short and modified version of the big five personality model. This finding, in contrast to other findings from the present study, suggests that the emotion regulation difficulty dimensions are more correlated with maladaptive personality rather than adaptive personality.

Although the present study contained valuable insights on the associations between different adaptive and maladaptive dimensions of personality with emotion regulation, which could be useful in distinguishing between personality models, the research also had limitations: First, the present study was conducted on a normal population, and it was cross-sectional; therefore, 
causal relationships cannot be mapped out. Secondly, different psychological, social, and cultural dimensions can affect the relationship between personality dimensions and the difficulty of emotional regulation, so intercultural and longitudinal studies as well as studies on clinical populations can lead to valuable results and insights.

\section{References}

1. Ali A, Toner BB, Stuckless N, et al. Emotional abuse, selfblame, and self-silencing in women with irritable bowel syndrome. Psychosom Med 2000; 62: 76-82.

2. Ali F, Amorim IS, Chamorro-Premuzic T. Empathy deficits and trait emotional intelligence in psychopathy and Machiavellianism. Pers Indiv Differ 2009; 47: 758-762.

3. American Psychiatric Association. Diagnostic and statistical manual of mental disorders (5 $5^{\text {th }}$ ed.). DC: American Psychiatric Publishing, Washington 2013.

4. American Psychiatric Association. Diagnostic and statistical manual of mental disorders (3 ${ }^{\text {rd }}$ ed.). DC: American Psychiatric Publishing, Washington 1980.

5. American Psychiatric Association. Diagnostic and statistical manual of mental disorders (4th ed.). DC: American Psychiatric Publishing, Washington 1994.

6. Azad-Marzabadi E, Amiri S. Morningness-eveningness and emotion regulation difficulty incremental validity in predicting social anxiety dimensions. Int I General Med 2017; 10: 275-279.

7. Betts J, Gullone E, Allen JS. An examination of emotion regulation, temperament, and parenting style as potential predictors of adolescent depression risk status: A correlational study. Br J Dev Psychol 2009; 27: 473-485.

8. Bolger N, Schilling EA. Personality and the problems of everyday life: The role of neuroticism in exposure and reactivity to daily stressors. J Personal 1991; 59: 355-386.

9. Bradley MM, Lang PJ. Emotion and motivation. In: Handbook of Psychophyiology $\left(2^{\text {nd }}\right.$ ed.). JT Cacioppo, LG Tassinary, GG Berntson (eds.). Cambridge University Press, Cambridge 2000; 602-642.

10. Brook M, Kosson DS. Impaired cognitive empathy in criminal psychopathy: Evidence from a laboratory measure of empathic accuracy. J Abnorm Psychol 2013; 122: 156-166.

11. Clark LA. Assessment and diagnosis of personality disorder: Perennial issues and emerging conceptualization. Ann Rev Psychol 2007; 58: 227-258.

12. Cole PM, Michel MK, Teti LO. The development of emotion regulation and dysregulation: A clinical perspective. Monogr Soc Res Child Dev 1994; 59: 73-100.

13. Corstorphine E. Cognitive-emotional-behavioural therapy for the eating disorders: working with beliefs about emotions. Europ Eat Dis Rev 2006; 14: 448-461

14. Cramer KM, Gallant MD, Langlois MW. Self-silencing and depression in women and men: Comparative structural equation models. Pers Indiv Differ 2005; 39: 581-592.

15. d'Acremont $M$, van der Linden $M$. How is impulsivity related to depression in adolescence? Evidence from a French validation of the Cognitive Emotion Regulation Questionnaire. J Adolesc 2007; 30, 271-282.

16. Dahl RE. Adolescent development and the regulation of behavior and emotion: Introduction to part VIII. Ann N Y Acad Sci 2004; 1021: 294-295.

17. Dasch KB, Cohen LH, Sahl JC, Gunthert KC. Moderating effects of sociotropy and autonomy on affective and self-esteem reactivity to daily stressors. Cogn Therapy Res 2008; 32: 177-195.

18. Donnellan MB, Oswald FL, Baird BM, Lucas RE. The MiniIPIP scales: Tiny-yet-effective measures of the big five factors of personality. Psychol Assess 2006; 18: 192-203.

19. Ehlers A, Clark DM. A cognitive model of posttraumatic stress disorder. Behav Res Ther 2000; 38: 319-345.

20. Fossati A, Somma A, Borroni S, Markon KE, Krueger RF. The Personality Inventory for DSM-5 Brief Form: Evidence for Reliability and Construct Validity in a Sample of Community-Dwelling Italian Adolescents. Assessment 2017; 24: 615-631.

21. George D, Mallery P. SPSS for Windows step by step: a simple guide and reference. 11.0 update ( $4^{\text {th }}$ ed.). Allyn and Bacon, Boston 2003.

22. Gratz KL, Roemer L. Multidimensional assessment of emotion regulation and dysregulation: Development, factor structure, and initial validation of the difficulties in emotion regulation scale. J Psychopathol Behav Assess 2004; 26: 41-54.

23. Gross JJ, Thompson RA. Emotion regulation: Conceptualfoundations. In: Handbook of emotion regulation. JJ Gross (ed.). Guilford Press, New York 2007; 3-24.

24. Gross J. The emerging field of emotion regulation: An integrative review. Rev Gen Psychol 1998; 2: 271-299.

25. Gross JJ, Levenson RW. Hiding feelings: The acute effects of inhibiting negative and positive emotion. J Abnorm Psychol 1997; 106: 95-103.

26. Harkness AR, McNulty IL, Ben-Porath YS. The Personality Psychopathology Five (PSY-5): Constructs and MMPI-2 scales. Psychol Assess 1995; 7: 104-114.

27. Herr NR, Rosenthal MZ, Geiger PJ, Erikson K. Difficulties with emotion regulation mediate the relationship between borderline personality disorder symptom severity and interpersonal problems. Personal Ment Health 2013; 7: 191-202.

28. Jakobwitz S, Egan V. The Dark Triad and normal personality traits. Pers Indiv Differ 2006; 40: 331-339.

29. Jonason PK, Lyons M, Bethell E, Ross R. Different routes to limited empathy in the sexes: Examining the links between the Dark Triad and empathy. Pers Indiv Differ 2013; 57: $572-576$

30. Jonason PK, Webster GD, Schmitt DP, Li NP, Crysel L. The antihero in popular culture: A life history theory of the Dark Triad. Rev Gen Psychol 2012; 16: 192-199.

31. Jones DN, Paulhus DL. Differentiating the Dark Triad within the interpersonal circumplex. In: Handbook of interpersonal psychology: Theory, research, assessment, and therapeutic interventions. LM Horowitz, S Strack (eds.). Wiley; New York 2011; 249-268.

32. Jones DN, Paulhus DL. Introducing the Short Dark Triad (SSD3): A brief measure of dark personality trait. Assessment 2014; 21: 28-41.

33. Kring A, Sloan D. Emotion regulation and psychopathology. Guilford Press; New York 2010.

34. Krueger RF, Derringer J, Markon KE, Watson D, Skodol AE. Initial construction of a maladaptive personality trait model and inventory for DSM-5. Psychol Med 2012; 42: 1879-1890.

35. Laverdière O, Morin AJS, St-Hilaire F. Factor structure and measurement invariance of a short measure of the Big Five personality traits. Pers Indiv Differ 2013; 55: 739-743.

36. Macklem GL. Practitioner's guide to emotion regulation in school-aged children. Springer-Verlag; New York 2008.

37. Malterer MB, Glass SJ, Newman JP. Psychopathy and trait emotional intelligence. Pers Indiv Differ 2008; 44: 735745. 
38. McCrae RR, Costa PT. Validation of thefive-factor model of personality across instruments and observers. J Pers Social Psychol 1987; 52: 81-90.

39. Mroczek DK, Almeida DM. The effect of daily stress, personality, and age on daily negative affect. J Pers 2004; 72: 355-378.

40. Panksepp P. What is an emotional feeling? Lessons about affective origins from cross-species neuroscience. Motiv Emot 2012; 36, 4-15.

41. Patrick CJ, Lang AR. Psychopathic traits and intoxicated states: Affective concomitants and conceptual links. In: Startle modification: Implications for neuroscience, cognitive science, and clinical science. ME Dawson, AM Schell, AH Boehmelt (eds.). Cambridge University Press, New York 1999.

42. Paulhus DL, Williams KM. The Dark Triad of personality: Narcissism, Machiavellianism, and psychopathy. J Res Pers 2002; 36: 556-563.

43. Pollock NC, McCabe GA, Ashton C. Southard, Virgil Zeigler-Hill. Pathological personality traits and emotion regulation difficulties. Pers Indiv Differ 2016; 95: 168-177.

44. Roberts BW, Walton KE, Veichtbauer W. Patterns of mean-level change in personality traits across the life course: A meta-analysis of longitudinal studies. Psychol Bull 2006; 132: 1-25.

45. Rosenthal MZ, Gratz KL, Kosson DS, Cheavens IS, Lejuez CW, Lynch TR. Borderline personality disorder and emotional responding: A review of the research literature. Clin Psychol Rev 2008; 28: 75-91.

46. Shamay-Tsoory SG, Aharon-Peretz J, Perry D. Two sys tems for empathy: A double dissociation between emotional and cognitive empathy in inferior frontal gyrus versus ventromedial prefrontal lesions. Brain 2009; 12: 617-627.

47. Surawy C, Hackmann A, Hawton K, Sharpe M. Chronic fatigue syndrome: a cognitive approach. Behav Res Ther 1995; 33: 535-544.

48. Timmermans T, Van Mechelen I, Nezlek JB. Individual differences in core affect reactivity. Pers Indiv Differ 2009; 47: 510-515.

49. Trull TJ, Durrett CA. Categorical and dimensional models of personality disorder. Ann Rev Clin Psychol 2005; 1: 355-380.

50. Twenge JM, Campbell KW. "Isn't it fun to get the respect that we're going to deserve?". Narcissism, social rejection, and aggression. Pers Soc Psychol Bull 2003; 29: 261-272.

51. Vuilleumier P. How brains beware: neural mechanisms of emotional attention. Trends Cogn Sci 2005; 9: 585-594.

52. Wai M, Tiliopoulos $N$. The affective and cognitive empathic nature of the dark triad of personality. Pers Indiv Differ 2012; 52: 794-799.

53. Widiger TA, Simonsen E. Alternative dimensional models of personality disorder: Finding a common ground. J Pers Disor 2005; 19: 110-130.

54. Woolfolk RL, Allen LA. Treating somatization: a cognitive-behavioural approach. Guilford Press, New York 2007. 